Size-dependent alternation of magnetoresistive properties in atomic chains

E. Durgun, R. T. Senger, H. Mehrez, H. Sevinçli, and S. Ciraci

Citation: The Journal of Chemical Physics 125, 121102 (2006); doi: 10.1063/1.2354080

View online: http://dx.doi.org/10.1063/1.2354080

View Table of Contents: http://aip.scitation.org/toc/jcp/125/12

Published by the American Institute of Physics

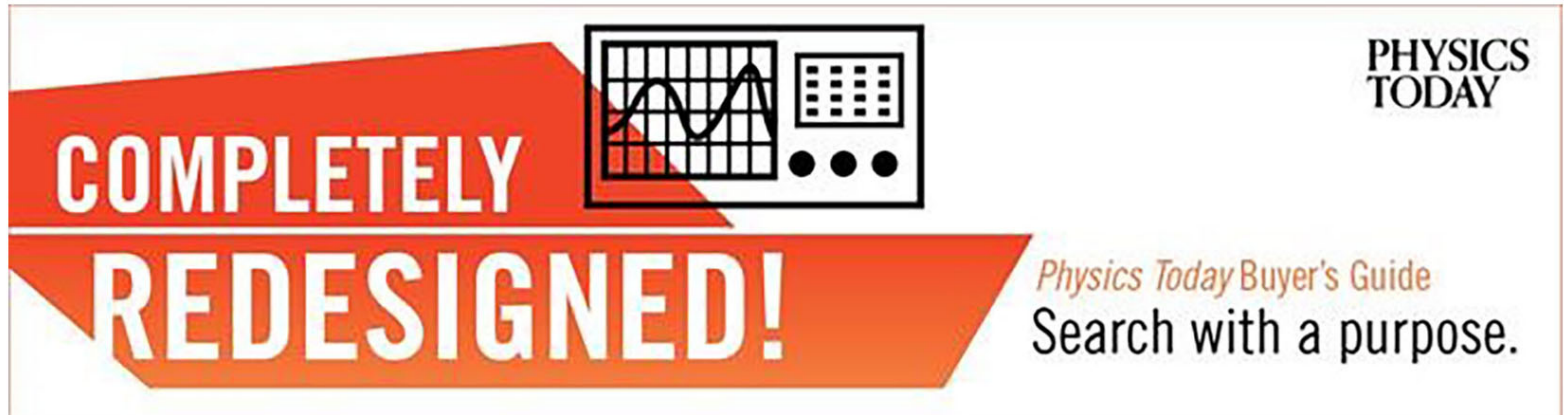




\title{
Size-dependent alternation of magnetoresistive properties in atomic chains
}

\author{
E. Durgun and R. T. Senger \\ Department of Physics, Bilkent University, Ankara 06800, Turkey \\ H. Mehrez \\ Center for NanoTechnology and NASA Advanced Supercomputing Division, NASA Ames Research Center, \\ Mail Stop 229-1, Moffett Field, California 94035-1000 \\ H. Sevinçli and S. Ciraci ${ }^{\text {a) }}$ \\ Department of Physics, Bilkent University, Ankara 06800, Turkey
}

(Received 18 April 2006; accepted 16 August 2006; published online 28 September 2006)

\begin{abstract}
Spin-polarized electronic and transport properties of carbon atomic chains are investigated when they are capped with magnetic transition-metal (TM) atoms like $\mathrm{Cr}$ or Co. The magnetic ground state of the TM- $\mathrm{C}_{n}$-TM chains alternates between the ferromagnetic $(\mathrm{F})$ and antiferromagnetic (AF) spin configurations as a function of $n$. In view of the nanoscale spintronic device applications the desirable AF state is obtained for only even- $n$ chains with Cr; conversely only odd- $n$ chains with Co have AF ground states. When connected to appropriate metallic electrodes these atomic chains display a strong spin-valve effect. Analysis of structural, electronic, and magnetic properties of these atomic chains, as well as the indirect exchange coupling of the TM atoms through non-magnetic carbon atoms are presented. (C) 2006 American Institute of Physics. [DOI: 10.1063/1.2354080]
\end{abstract}

Typical devices based on giant magnetoresistance effect ${ }^{1}$ are made of magnetic multilayers, where the relative alignment of the layer magnetizations causes large variations in the resistance of the structure. In such magnetic superlattice structures, the interlayer exchange coupling and the magnetoresistance are found to be oscillating as a function of spacer thickness. ${ }^{2-4}$ Recently, efforts in combining spintronics with molecular electronics have focused on lower dimensional analogs of the layer magnetic heterostructures. Along this direction, spin-polarized electronic transport in molecular devices has been investigated extensively. ${ }^{5-11}$ Organic and carbon-based molecules are promising in achieving magnetoresistive effects. Linear atomic chains of $\mathrm{C}$ have been synthesized, ${ }^{12}$ and their impressive properties have been demonstrated. ${ }^{13}$ Incorporation of transition-metal (TM) atoms into a $\mathrm{C}$ linear chain in the form of impurities, and formation of infinite periodic compound structures are energetically feasible. Such infinite, periodic $\left(\mathrm{TM}-\mathrm{C}_{n}\right)$ compound structures are found to be stable and half-metallic. ${ }^{14}$ Pati et $a l .{ }^{15}$ have studied atomic wires consisting of $\mathrm{C}$ and $\mathrm{Co}$ atoms where they have concluded that the ground state of short $\mathrm{CoC}_{n} \mathrm{Co}$ atomic chains is antiferromagnetic.

In this Communication, we find that the isolated TM- $C_{n}$-TM atomic chains or linear molecules (TM denoting $\mathrm{Co}, \mathrm{Cr}, \mathrm{Fe}$, or $\mathrm{Mo}$ ) alternate between ferromagnetic (F) and antiferromagnetic $(\mathrm{AF})$ ground states as a function of the number of $\mathrm{C}$ atoms $n$. Changing the value of $n$ by one modifies the magnetic ground state from $\mathrm{F}$ to $\mathrm{AF}$, or vice versa. Moreover, whether even- or odd- $n$ leads to an AF ground state depends on the type of the TM atom. We also find that the generic electronic properties of these chains are preserved after attaching them to appropriate semi-infinite leads

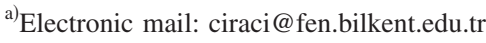

at both ends. Hence, they act as almost perfect spin valves when they are in a F state.

The oscillatory indirect exchange interaction (known as RKKY) between localized magnetic moments, mediated by the itinerant carriers of a spacer medium, occurs in extended metallic hosts. It is a second order perturbative effect that plays a significant role in determining the magnetic order in low-dimensional structures. Here, owing to the zerodimensional nature and hence finite level spacing of the TM- $\mathrm{C}_{n}$-TM structures and non-perturbative character of the interaction of the TM atoms with the carbon chain an RKKYtype formulation is not appropriate. Rather, one needs to employ self-consistent density functional methods or a direct diagonalization of the spin-dependent model Hamiltonian of the system.

We have performed first-principles plane wave calculations ${ }^{16}$ within density functional theory (DFT) using ultra-soft pseudopotentials. ${ }^{17}$ The exchange correlation potential has been approximated by generalized gradient approximation. ${ }^{18}$ The atomic positions of the structures are optimized by minimizing the total energy as well as the forces on the atoms using spin-relaxed calculations. ${ }^{19}$ The results of plane-wave calculations are compared and confirmed with methods which utilize local-basis sets. ${ }^{20,21}$ Transition state analysis performed for different reaction paths, extensive stability analysis, and $a b$ initio molecular dynamic calculations performed at $1000 \mathrm{~K}$ show that $\mathrm{CoC}_{n} \mathrm{Co}$ and $\mathrm{CrC}_{n} \mathrm{Cr}$ isolated atomic chains can be synthesized and are stable. For example, both $\mathrm{CoC}_{n} \mathrm{Co}$ and $\mathrm{CrC}_{n} \mathrm{Cr}$ isolated chains can be formed from a finite, linear $\mathrm{C}_{n}$ chain by attaching $\mathrm{Co}$ or $\mathrm{Cr}$ atoms to both ends. ${ }^{14}$

The calculated electronic and magnetic properties of isolated $\mathrm{CrC}_{n} \mathrm{Cr}$ and $\mathrm{CoC}_{n}$ Co chains are summarized in Table $\mathrm{I}$. The total energy of every structure is calculated for each 
TABLE I. The calculated electronic properties of isolated $\mathrm{CrC}_{n} \mathrm{Cr}$ and $\mathrm{CoC}_{n} \mathrm{Co}$ chains using vaSP (Ref. 16). The columns are respectively: The energy difference of the $\mathrm{AF}$ and the lowest-energy $\mathrm{F}$ states, $\Delta E_{F \rightarrow A F}$ $=E_{T}(A F)-E_{T}(F)$, magnetic moment $\mu$ of the ground state in units of Bohr magneton $\mu_{B}$ (for $\mathrm{AF}$ ground states the moment corresponding to the higher energy $\mathrm{F}$ state is given in parenthesis), $E_{g}^{\sigma}$ are the energy gap between the highest occupied and lowest unoccupied levels of spin- $\sigma$ states. The bold numbers denote the spin type of the HOMO levels.

\begin{tabular}{|c|c|c|c|c|}
\hline Structure & $\Delta E_{F \rightarrow A F}(\mathrm{eV})$ & $\mu\left(\mu_{B}\right)$ & $E_{g}^{\uparrow}(\mathrm{eV})$ & $E_{g}^{\downarrow}(\mathrm{eV})$ \\
\hline $\mathrm{CrCCr}$ & 1.12 & 8 & 0.97 & 2.37 \\
\hline $\mathrm{CrC}_{2} \mathrm{Cr}$ & -0.10 & $0(10)$ & $1.43(\mathbf{0 . 8 2})$ & $1.43(3.56)$ \\
\hline $\mathrm{CrC}_{3} \mathrm{Cr}$ & 0.87 & 8 & 1.33 & 1.83 \\
\hline $\mathrm{CrC}_{4} \mathrm{Cr}$ & -0.08 & $0(10)$ & $1.26(\mathbf{0 . 9 1})$ & $1.26(2.97)$ \\
\hline $\mathrm{CrC}_{5} \mathrm{Cr}$ & 0.70 & 8 & 1.43 & 1.79 \\
\hline $\mathrm{CrC}_{6} \mathrm{Cr}$ & -0.06 & $0(10)$ & $1.17(\mathbf{0 . 8 8})$ & 1.17 (2.77) \\
\hline $\mathrm{CrC}_{7} \mathrm{Cr}$ & 0.58 & 8 & 1.32 & 1.63 \\
\hline $\mathrm{CoCCo}$ & -0.17 & $0(2)$ & $0.24(1.41)$ & $0.24(\mathbf{0 . 4 0})$ \\
\hline $\mathrm{CoC}_{2} \mathrm{Co}$ & 0.32 & 4 & 1.80 & 0.55 \\
\hline $\mathrm{CoC}_{3} \mathrm{Co}$ & -0.12 & $0(2)$ & $0.87(1.67)$ & $0.87(\mathbf{0 . 8 6})$ \\
\hline $\mathrm{CoC}_{4} \mathrm{Co}$ & 0.28 & 4 & 1.72 & 0.26 \\
\hline $\mathrm{CoC}_{5} \mathrm{Co}$ & -0.13 & $0(2)$ & $0.88(1.48)$ & $0.88(\mathbf{0 . 8 5})$ \\
\hline $\mathrm{CoC}_{6} \mathrm{Co}$ & 0.24 & 4 & 1.62 & 0.48 \\
\hline $\mathrm{CoC}_{7} \mathrm{Co}$ & -0.13 & $0(2)$ & $0.79(1.34)$ & $0.79(\mathbf{0 . 7 5})$ \\
\hline
\end{tabular}

possible value of its magnetic moment $\mu$. The $\mathrm{AF}$ state $(\mu$ $=0$ ) arises as the ground state of the $\mathrm{CrC}_{n} \mathrm{Cr}$ chains only for even $n$. In this case, the state with $\mu=10 \mu_{B}$ is the lowestenergy $\mathrm{F}$ configuration. Chains with odd $n$, however, have a $\mathrm{F}$ ground state with $\mu=8 \mu_{B}$. Therefore, the quantity $\Delta E_{F \rightarrow A F}=E_{T}(A F)-E_{T}(F)$, defined as the energy difference of the $\mathrm{AF}$ and the lowest $\mathrm{F}$ states of the structures, displays an alternating variation with $n$. Interestingly, a similar alternation for $\Delta E_{F \rightarrow A F}$ in $\mathrm{CoC}_{n}$ Co chains is found with an inverted form; the AF ground state is obtained only for odd- $n$ cases as seen in Fig. 1. The variation of $\Delta E_{F \rightarrow A F}$ in chains with terminal $\mathrm{Mo}$ and $\mathrm{Fe}$ atoms resembles to the $\mathrm{Cr}$ and Co cases, respectively. On the other hand, with $\mathrm{Ti}$ or $\mathrm{Sc}$, for instance, such regular alternations are not found. Apparently, the electronic configuration of the outer $d$-orbitals of the TM atoms

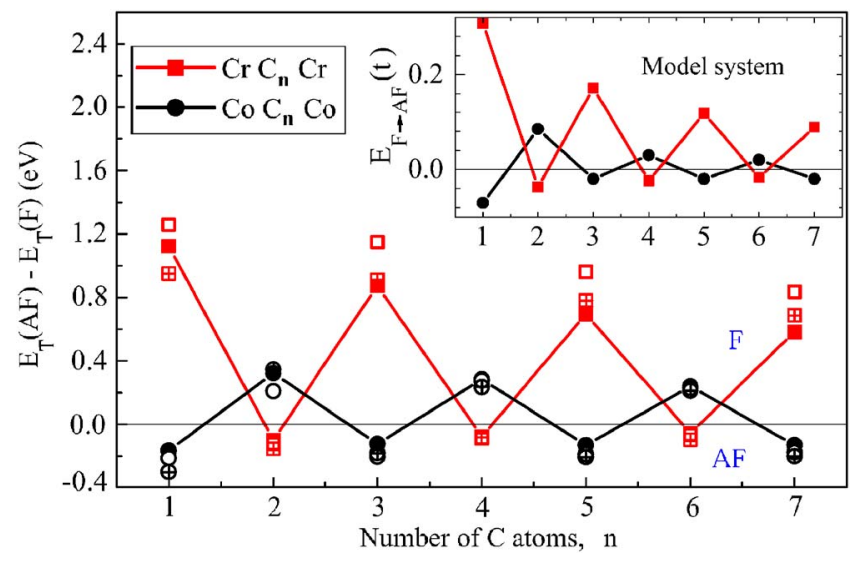

FIG. 1. (Color online) Energy difference of the $\mathrm{AF}$ and the $\mathrm{F}$ states in the $\mathrm{CrC}_{n} \mathrm{Cr}$ and $\mathrm{CoC}_{n} \mathrm{Co}$ atomic chains as a function of the number of $\mathrm{C}$ atoms. The ground state is AF for negative values of the energy difference. The solid, open, and crossed symbols represent the results of VASP, ${ }^{16}$ SIESTA, ${ }^{20}$ and GAUSSIANO $3^{21}$ calculations, respectively. The inset shows similar variations obtained in a model system.

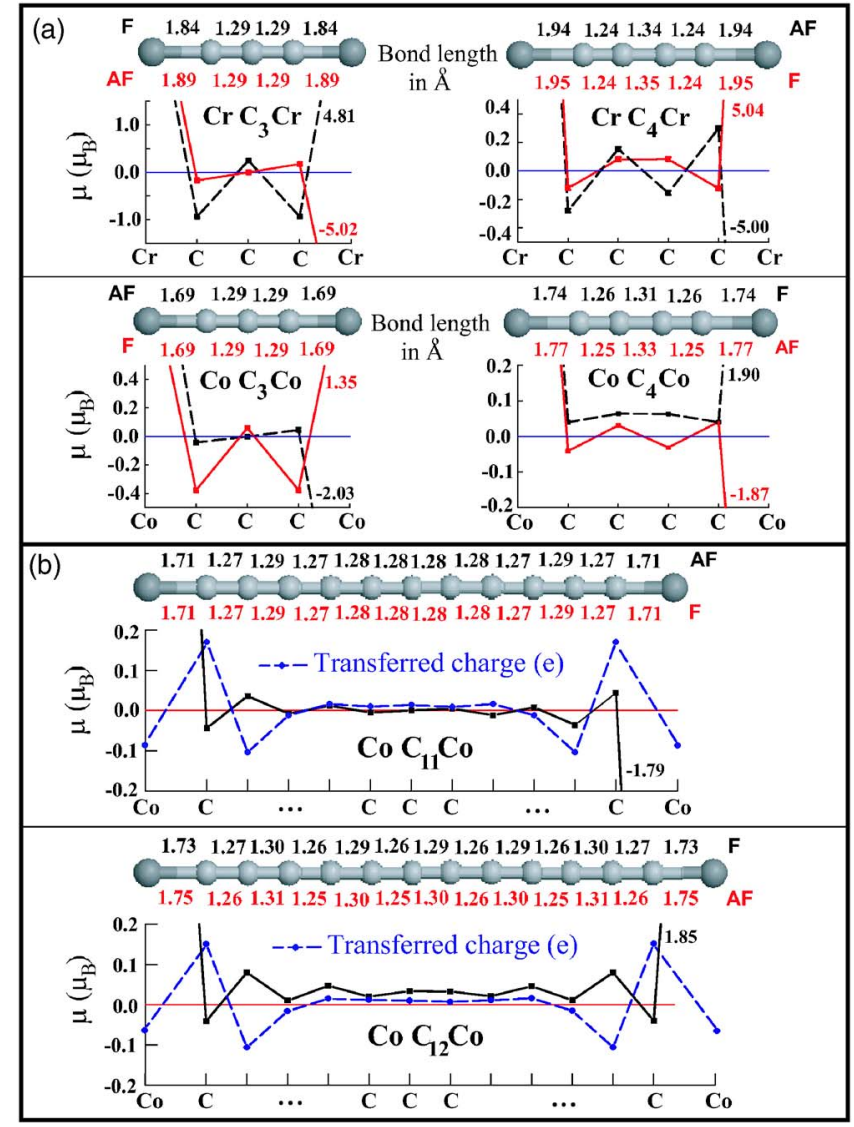

FIG. 2. (Color online) (a) Molecular structure and atomic magnetic moments in isolated $\mathrm{CrC}_{n} \mathrm{Cr}$ and $\mathrm{CoC}_{n}$ Co chains for $n=3$ and 4 in their $\mathrm{AF}$ and $\mathrm{F}$ states. The values of the moments on the TM atoms are also denoted. (b) Same for longer chains. The charge transfer and induced atomic moments are plotted only in the ground states.

is essential in the form of the carbon-mediated interaction between them.

The quantity $\Delta E_{F \rightarrow A F}$ is related to the strength of the indirect exchange interaction between the TM atoms. It is the energy required to invert the magnetic moment of one of the $\mathrm{TM}$ atoms in the $\mathrm{F}$ state. The situation bears some resemblance to the case of indirect exchange coupling of two Co adatoms on a metallic carbon nanotube. Costa et al. ${ }^{7}$ have obtained an oscillatory variation of the exchange coupling between the Co adatoms as a function of their separation. The indirect exchange interaction was extremely longranged, and the period of oscillations was larger than the structural period of the carbon nanotube. In our case, however, the period of alternation of magnetic ground state is shorter; its type is altered when the number of $\mathrm{C}$ atoms is changed by one. It is the $\mathrm{C}_{n}$ atomic chain that mediates the interaction in an involved manner. In the F and AF states, the variation of the induced magnetic moments on the $\mathrm{C}$ atoms changes, and the atomic structures of the molecules are also slightly modified.

In Fig. 2, we display the equilibrium atomic structure and the variation of atomic magnetic moments in $\mathrm{CrC}_{n} \mathrm{Cr}$ $(n=3,4)$, and $\mathrm{CoC}_{n}$ Co chains $(n=3,4,11,12)$ as representative samples. In the even- $n$ cases the $\mathrm{C}$ atoms tend to dimerise, whereas for odd- $n$ the $\mathrm{C}-\mathrm{C}$ bonds are almost uniform (this property is more evident for larger $n$ cases). The atomic 
magnetic moments are calculated based on an orbitalresolved Mulliken analysis. ${ }^{20}$ The magnetic TM atoms induce moments throughout the $\mathrm{C}$ chain which set up an indirect exchange interaction between them. Owing to the chain geometry and quantum interference effects, the induced magnetization of $\mathrm{C}$ atoms is quite long-ranged; for instance, in the $\mathrm{F}$ ground state of the $\mathrm{Cr}_{15} \mathrm{Cr}$ structure all the $\mathrm{C}$ atoms have alternating magnetic moments of $|\mu| \sim 0.1 \mu_{B}$. The effect is somewhat weaker for the Co cases; in Fig. 2(b) the chains with $n=11$ and 12 have higher induced charge and magnetic moments on the two nearest-neighbor $\mathrm{C}$ atoms, but they get damped deeper in the chain. Our results are in accord with a recent experimental study on contact induced magnetism in metallic carbon nanotubes, ${ }^{22}$ where they find that spin-polarized charge transfer at the interface between a flat ferromagnetic metal substrate $(\mathrm{Co})$ and a multiwalled carbon nanotube leads to a spin transfer of about $0.1 \mu_{B}$ per contact $\mathrm{C}$ atom.

The qualitative features of the peculiar magnetic ordering in these chain structures can be understood through a Hückel-type "toy" model. Consider the Hamiltonian of a chain structure having $n+2$ sites: $H=\Sigma_{i, \sigma} \varepsilon_{i, \sigma} c_{i, \sigma}^{\dagger} c_{i, \sigma}$ $-\Sigma_{i, \sigma}\left(t_{i, i+1 ; \sigma} c_{i, \sigma}^{\dagger} c_{i+1, \sigma}+\right.$ h.c. $)$, where $\varepsilon_{i, \sigma}$ is local site energy, $t_{i, i+1 ; \sigma}$ is the spin-dependent nearest-neighbor hopping integral, $c_{i, \sigma}^{\dagger}$ and $c_{i, \sigma}$ denote fermion creation and annihilation operators for site $i$ (from 0 to $n+1$ ) with spin $\sigma$, respectively. The nonmagnetic sites $(i=1$ to $n$ ) are represented by spindegenerate on-site energies of zero, and $t_{i, i+1 ; \sigma}=t$. To mimic the magnetic sites $(i=0$ and $i=n+1)$, however, we take both the on-site energies and their coupling to nearest nonmagnetic sites as spin-dependent: $\varepsilon_{0, \uparrow}=\epsilon_{1}$ and $\varepsilon_{0, \downarrow}=\epsilon_{2} ; t_{0,1 ; \uparrow}=t_{1}$ and $t_{0,1 ; \downarrow}=t_{2}$. For the sake of simplicity, we model the magnetic sites with only two spin-dependent states. In the F state of the molecules the two magnetic sites are equivalent, and the AF configuration can be represented by taking one of the magnetic sites $(i=n+1)$ to have on-site energies and hopping terms with inverted spin labels: $\varepsilon_{n+1, \uparrow}=\epsilon_{2}, \quad \varepsilon_{n+1, \downarrow}=\epsilon_{1}$, $t_{n, n+1 ; \uparrow}=t_{2}$, and $t_{n, n+1 ; \downarrow}=t_{1}$. Diagonalizing the Hamiltonian, the total energy of the molecule is calculated at half-filling for the F and AF configurations separately. Depending on the parameter set $\left\{\epsilon_{1}, \epsilon_{2}, t_{1}, t_{2}\right\}$ the variation of the total energy difference $\Delta E_{F \rightarrow A F}$ with $n$ shows several distinct patterns, including the type of alternations compatible with the DFT calculations of the $\mathrm{Cr}$ and $\mathrm{Co}$ cases. For instance, the sets $\{0.0,-1.0,1.0,1.5\}$ and $\{-0.3,-1.0,0.5,1.5\}$ in units of $t$ reproduce $\mathrm{Cr}$ - and Co-like variations, respectively (see Fig. 1). The model is capable of simulating the DFT results, since it can mimic the dominant mechanisms of charge and spin induction on the nonmagnetic spacer, as well as the quantum interference effects. The magnetic TM atoms certainly have strong spin polarizations justifying different on-site energies for electrons of each spin type in the model. That asymmetry is expected to be stronger for $\mathrm{Cr}$ than for Co due to Hunds rule. When the TM atoms are in contact with the nonmagnetic $\mathrm{C}$ chain, the couplings of the TM spin-dependent states with the carbon states are also different. The resulting effect is to create a charge and spin polarization on the $\mathrm{C}$ network which also sets up the indirect exchange interaction between the TM atoms. The analysis of the DFT results show that not

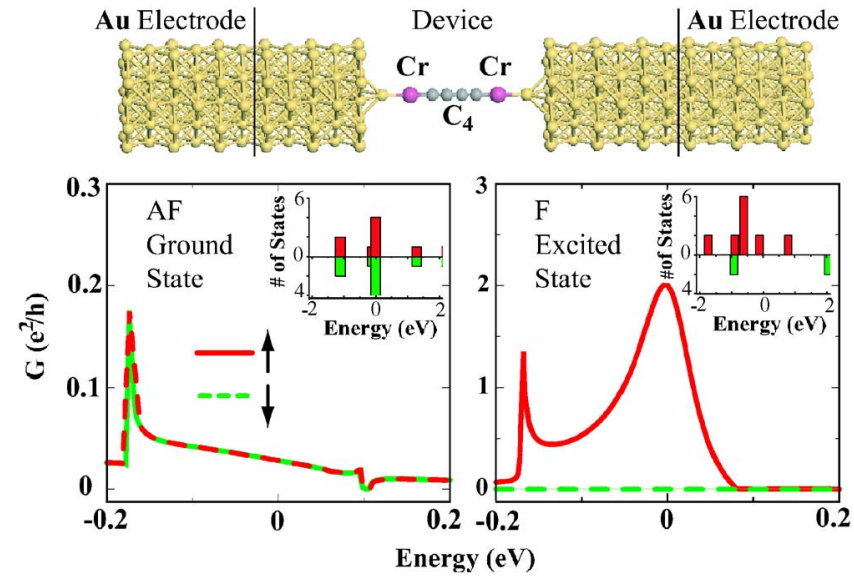

FIG. 3. (Color online) Spin polarized conductance of $\mathrm{CrC}_{4} \mathrm{Cr}$ molecular spin-valve device when connected to semi-infinite Au electrodes. The insets show the molecular energy levels of the isolated chains.

only the $\pi$-conjugated states of the carbon chain but also the lower-energy $\sigma$-bonding states contribute significantly to the indirect interaction.

Conductance properties of molecular devices depend not only on their intrinsic structure but also on the electrodes. In particular, if the coupling of the device to the electrodes is strong, the electronic structure and hence the transport properties of the device can be quite different, and cannot be inferred from the electronic structure of the isolated device. We have examined $\mathrm{Au}$ and $\mathrm{Al}$ atomic chains, and $\mathrm{Au}$ leads with finite cross section to model the electrodes. The TM- $\mathrm{C}_{n}-\mathrm{TM}$ structures having an $\mathrm{AF}$ ground state remained AF with smaller $\left|\Delta E_{F \rightarrow A F}\right|$ values in cases of $\mathrm{Au}$ electrodes, whereas the ground states were changed to F upon connecting to $\mathrm{Al}$ electrodes. Here, we choose to discuss the case of finite cross-section $\mathrm{Au}$ electrodes with single-atom sharp tips, which couples weakly to the device and preserves the generic electronic structure of the isolated TM-C ${ }_{n}-\mathrm{TM}$ chains. Consequently, we obtain a prominent spin-valve effect that is compatible with the spin-relaxed energy configurations of the isolated molecules.

To calculate the transport characteristics, the effective device region with a long buffer of Au layers (see Fig. 3) is first solved within periodic boundary condition for both AF and $\mathrm{F}$ states. We considered the $\mathrm{Cr}$ cases for $n=4$ and 6 , and found that the ground state is $\mathrm{AF}$, and the $\mathrm{F}$ state has $\mu$ $=10 \mu_{B}$, same as in the case of isolated chains. For the Co cases, the chains studied for $n=3$ and 5 also preserved their AF ground states when attached to the Au leads. The selfconsistent calculations lead to spin-up and spin-down Hamiltonians, ${ }^{20}$ which are used to calculate the transmission coefficient for each spin state in the AF as well as in the higher energy F configurations. The surface Green's function of the contacts is calculated recursively. Following this, we calculate the conductance of the device within Landauer formalism, $G(E)=\left(e^{2} / h\right) \operatorname{Tr}\left(\boldsymbol{\Gamma}^{L} \mathbf{G}^{r} \boldsymbol{\Gamma}^{R} \mathbf{G}^{a}\right)$, for each spin configuration. ${ }^{23}$ In Fig. 3, we show our results for the $\mathrm{CrC}_{4} \mathrm{Cr}$ molecule. In the AF state of the device, the conductance of majority-spin (spin-up) electrons coincides with that of minority-spin (spin-down). However, if the system is in the excited $\mathrm{F}$ state, the conductance through the spin-down lev- 
els is substantially reduced while it is enhanced through the spin-up levels. The equilibrium conductance value is enhanced by a factor of $\sim 60$ for the $\mathrm{CrC}_{4} \mathrm{Cr}$ structure. This is a clear indication of a strong spin-valve effect through the TM- $\mathrm{C}_{n}$-TM molecular structures. Consistent with the energy levels of the isolated chain given in the insets of Fig. 3, it is the spin-up $\mathrm{HOMO}$ level in $\mathrm{CrC}_{4} \mathrm{Cr}$ that dominantly contributes to the conduction.

We should note that Pati et al. ${ }^{15}$ have also studied $\mathrm{CoC}_{n} \mathrm{Co}$ atomic chains, predicting that the ground state of these isolated structures are AF for all $n=1-15$ (except $n$ =14). However, the alternating variation found in this study is novel and dramatically different than theirs. We believe that in the calculations of Ref. 15, the true ground states of the structures have not been found. The present results concerning with the alternating magnetic ground states of TM- $\mathrm{C}_{n}$-TM chains have been consistently reproduced by the calculations using three independent codes. ${ }^{16,20,21}$ Furthermore, they have been explained successfully by a simple analytical model that produces the same F-AF alternations of the ground states (see Fig. 1).

In summary, we find that the magnetic ground state of TM-atom-capped $\mathrm{C}$ atomic chains alternates between $\mathrm{AF}$ and $\mathrm{F}$ states depending on the number of $\mathrm{C}$ atoms in the chain. The indirect exchange interaction of the TM atoms is mediated by the induced spin polarizations on the $\mathrm{C}$ atoms which is due to the asymmetric coupling of the spin states of the $\mathrm{TM}$ atoms with the $\mathrm{C}$ states. Furthermore, we showed that an almost perfect spin-valve effect is present in these simple molecular structures. These properties can be engineered by selecting the type of the TM atom and the number of $\mathrm{C}$ atoms in the chain which lead to odd-even disparities in their magnetic and transport properties. These impressive characteristics are of fundamental and technological interest in the promising fields of spintronics and molecular electronics.

R.T.S. acknowledges partial financial support from TÜBA-GEBIP.

${ }^{1}$ M. N. Baibich, J. M. Broto, A. Fert, F. Nguyen Van Dau, F. Petroff, P. Etienne, G. Creuzet, A. Friedrich, and J. Chazelas, Phys. Rev. Lett. 61, 2472 (1988); G. Binasch, P. Grünberg, F. Saurenbach, and W. Zinn, Phys. Rev. B 39, 4828 (1989).

${ }^{2}$ S. S. P. Parkin, N. More, and K. P. Roche, Phys. Rev. Lett. 64, 2304 (1990).
${ }^{3}$ D. M. Edwards, J. Mathon, R. B. Muniz, and M. S. Phan, Phys. Rev. Lett. 67, 493 (1991).

${ }^{4}$ P. Bruno, Phys. Rev. B 52, 411 (1995).

${ }^{5}$ M. S. Ferreira and S. Sanvito, Phys. Rev. B 69, 035407 (2004).

${ }^{6}$ W. I. Babiaczyk and B. R. Bulka, J. Phys.: Condens. Matter 16, 4001 (2004).

${ }^{7}$ A. T. Costa, Jr., D. F. Kirwan, and M. S. Ferreira, Phys. Rev. B 72, 085402 (2005).

${ }^{8}$ E. G. Emberly and G. Kirczenow, Chem. Phys. 281, 311 (2002).

${ }^{9}$ Y. Wei, Y. Zu, J. Wang, and H. Guo, Phys. Rev. B 70, 193406 (2004).

${ }^{10}$ A. R. Rocha, V. M. García-Suárez, S. W. Bailey, C. J. Lambert, J. Ferrer, and S. Sanvito, Nat. Mater. 4, 335 (2005); A. R. Rocha, V. M. GarcíaSuárez, S. Bailey, C. Lambert, J. Ferrer, and S. Sanvito, Phys. Rev. B 73, 085414 (2006).

${ }^{11}$ H. Dalgleish and G. Kirczenow, Phys. Rev. B 72, 184407 (2005).

${ }^{12}$ G. Roth and H. Fischer, Organometallics 15, 5766 (1996); X. Zhao, Y. Ando, Y. Liu, M. Jinno, and T. Suzuki, Phys. Rev. Lett. 90, 187401 (2003); S. Eisler, A. D. Slepkov, E. Elliott, T. Luu, R. McDonald, F. A. Hegmann, and R. R. Tykwinski, J. Am. Chem. Soc. 127, 2666 (2005).

${ }^{13}$ S. Tongay, R. T. Senger, S. Dag, and S. Ciraci, Phys. Rev. Lett. 93, 136404 (2004); S. Tongay, S. Dag, E. Durgun, R. T. Senger, and S. Ciraci, J. Phys.: Condens. Matter 17, 3823 (2005); R. T. Senger, S. Tongay, S. Dag, E. Durgun, and S. Ciraci, Phys. Rev. B 71, 235406 (2005).

${ }^{14}$ S. Dag, S. Tongay, T. Yildirim, E. Durgun, R. T. Senger, C. Y. Fong, and S. Ciraci, Phys. Rev. B 72, 155444 (2005).

${ }^{15}$ R. Pati, M. Mailman, L. Senapati, P. M. Ajayan, S. D. Mahanti, and S. K. Nayak, Phys. Rev. B 68, 014412 (2003); L. Senapati, R. Pati, M. Mailman, and S. K. Nayak, Phys. Rev. B 72, 064416 (2005).

${ }^{16}$ Most of the numerical computations have been carried out by using VASP software: G. Kresse and J. Hafner, Phys. Rev. B 47, 558 (1993); G. Kresse and J. Furthmüller, Phys. Rev. B 54, 11169 (1996).

${ }^{17}$ D. Vanderbilt, Phys. Rev. B 41, R7892 (1990).

${ }^{18}$ J. P. Perdew, J. A. Chevary, S. H. Vosko, K. A. Jackson, M. R. Pederson, and D. J. Singh, Phys. Rev. B 46, 6671 (1992).

${ }^{19}$ All molecular chain structures have been treated in tetragonal supercells using periodic boundary conditions. A large vacuum spacing $(\sim 10 \AA)$ has been taken in all directions. k-point sampling of the TM- $\mathrm{C}_{n}$-TM molecules is performed at the $\Gamma$ point. A plane-wave basis set with kinetic energy cutoff of $350 \mathrm{eV}$ has been used. The energy tolerance for selfconsistency is taken as $10^{-6} \mathrm{eV}$ and the maximum force allowed on each atoms is $0.05 \mathrm{eV} / \AA$.

${ }^{20}$ P. Ordejón, E. Artacho, and J. M. Soler, Phys. Rev. B 53, R10441 (1996); J. M. Soler, E. Artacho, J. D. Gale, A. Garcia, J. Junquera, P. Ordejón, and D. Sanchez-Portal, J. Phys.: Condens. Matter 14, 2745 (2002).

${ }^{21}$ M. J. Frisch, G. W. Trucks, H. B. Schlegel et al., GAUSSIAN 03, Revision C. 02, Gaussian Inc., Wollingford CT, 2004. The UBPW91 functional with $6-31 \mathrm{G}^{* *}$ basis set has been employed. The results are subjected to wave function stability analysis.

${ }^{22}$ O. Céspedes, M. S. Ferreira, S. Sanvito, M. Kociak, and J. M. D. Coey, J. Phys.: Condens. Matter 16, L155 (2004).

${ }^{23}$ S. Datta, Electron Transport in Mesoscopic Systems (Cambridge University Press, Cambridge, 1997). 